\title{
Heterozygote Expression in Propionyl Coenzyme A Carboxylase Deficiency
}

\section{DIFFERENCES BETWEEN MAJOR COMPLEMENTATION GROUPS}

\author{
BARRY WOlF and LeON E. Rosenberg, Departments of Human Genetics and \\ Pediatrics, Yale University School of Medicine, New Haven, Connecticut 06510
}

\begin{abstract}
A в S T R A C T We measured propionyl coenzyme A carboxylase (PCC) activity in extracts of skin fibroblasts and peripheral blood leukocytes from controls and obligate heterozygotes for PCC deficiency. 6 heterozygotes were from the $p c c$ A complementation group; 12 were from the other major complementation group, designated pcc C. Mean PCC activity in fibroblast extracts from $p c c A$ heterozygotes was $52 \%$ of that in controls, whereas mean PCC activity in pcc $C$ heterozygotes was indistinguishable from that of controls. Similar results were obtained with extracts of peripheral blood leukocytes. In none of eight families (three $p c c A$ and five $p c c C$ ) in which PCC activity was studied in both parents of an affected child were significant intrafamilial differences observed. The activities of two other mitochondrial enzymes ( $\beta$-methylcrotonyl CoA carboxylase and glutamate dehydrogenase) were comparable in controls and both groups of heterozygotes. Whereas the data from pcc A heterozygotes are consistent with expected gene dosage effects, those from $p c c C$ heterozygotes are not. Inasmuch as mammalian PCC is a large molecular weight tetramer, each protomer of which is probably composed of two nonidentical subunits, the latter results are most consistent with unbalanced rates of synthesis and(or) degradation of the two subunits in normal cells with compensatory balancing in pcc $C$ heterozygotes.
\end{abstract}

This work was presented in part at the annual meeting of the American Pediatric Society and the Society for Pediatric Research, 26-28 April 1978 in New York.

Dr. Wolf s address is the Departments of Human Genetics and Pediatrics, Medical College of Virginia, Richmond, Va. 23298. Dr. Wolf was a recipient of a traineeship from National Institutes of Health grant GM 00077.

Received for publication 12 June 1978 and in revised form 17 July 1978.

\section{INTRODUCTION}

Examination of all reported pedigrees containing at least 1 proband with propionic acidemia due to propionyl coenzyme A carboxylase (PCC) ${ }^{1}$ deficiency $(1,2)$, as well as those from 16 additional families known to the authors, indicates that PCC deficiency is inherited as an autosomal recessive trait. Past studies of obligate heterozygotes for this disorder have been limited. PCC activity in extracts of cultured skin fibroblasts from both parents of the first reported patient was approximately $50 \%$ of that in controls (3), suggesting that expression in obligate heterozygotes would conform to expected gene dosage effects. In the only other report in which obligate heterozygotes were examined, however, PCC activity in peripheral blood leukocytes from both parents of an affected child was within the control range (4). Recently, Gravel et al. (5) demonstrated two major genetic complementation groups among fibroblast lines from PCC-deficient patients and designated them the $p c c A$ and $p c c C$ groups. To determine whether the previous disparate observations in heterozygotes for PCC deficiency reflect this genetic heterogeneity, we have measured PCC activity in extracts of cultured fibroblasts and peripheral blood leukocytes from 18 obligate heterozygotes for PCC deficiency. We find that each of six heterozygotes from the pcc A complementation group have PCC activities approximately half that of controls, whereas all 12 heterozygotes from the $p c c C$ group have activities indistinguishable from controls.

\section{METHODS}

Cultured fibroblasts, initiated from skin biopsies, were propagated from 8 normal individuals, 10 children with PCC defi-

\footnotetext{
${ }^{1}$ Abbreviations used in this paper: $\mathrm{CoA}$, coenzyme $\mathrm{A} ; \boldsymbol{\beta}$ MCC, $\beta$-methylcrotonyl CoA carboxylase; PCC, propionyl CoA carboxylase; pcc A $(C)$, complementation group.
} 
ciency, and 18 parents and 1 sibling of PCC-deficient patients according to methods described previously (6). Cell lines from each PCC-deficient proband were placed in either the pcc $A$ or pcc $C$ group by genetic complementation studies $(5,7)$; lines from their respective parents were assumed to belong to the proband's complementation group. When defined this way, 6 obligate heterozygotes (parents) and 1 possible heterozygote (a sibling) from 3 families were from the pcc A group, and 12 obligate heterozygotes from 7 families (parents) were from the pcc $C$ group. Peripheral blood leukocytes, isolated from fresh heparinized blood as described previously (8), were obtained from 10 normal adults, 4 pcc $A$ obligate heterozygotes from 2 families, and 6 pcc $C$ obligate heterozygotes from 3 families.

PCC activity was measured in freshly harvested extracts of cultured fibroblasts and leukocytes as described previously (6). $\beta$-Methylcrotonyl CoA carboxylase ( $\beta$ MCC) activity was assayed in the same fibroblast extracts by substituting equimolar concentrations of $\beta$-methylcrotonyl $\operatorname{CoA}$ for propionyl CoA in the incubation mixture (9). Carboxylasespecific activities are expressed as picomole ${ }^{14} \mathrm{CO}_{2}$ fixed per minute per milligram of extract protein. Glutamate dehydrogenase was measured by a modification of a procedure described previously (10). Grouped enzyme activities are re- ported as the mean \pm 1 SD. Protein was determined by a modification of the method described by Lowry et al. (11).

\section{RESULTS}

Residual PCC activity in cells from probands. As noted in Fig. 1, some PCC activity was detected in all fibroblast extracts from patients with PCC deficiency. This residual activity was comparable in cells from both complementation groups, and ranged from 0.5 to $8 \%$ of control activity $(863 \pm 102 \mathrm{pmol} / \mathrm{min}$ per $\mathrm{mg}$ ). The activity of $\beta \mathrm{MCC}$, another mitochondrial biotin-dependent enzyme, was very similar in extracts from pcc $A$ homozygotes $(267 \pm 27 \mathrm{pmol} / \mathrm{min}$ per $\mathrm{mg})$, pcc $C$ homozygotes $(279 \pm 40 \mathrm{pmol} / \mathrm{min}$ per $\mathrm{mg})$ and controls $(273 \pm 37 \mathrm{pmol} / \mathrm{min}$ per $\mathrm{mg})$.

Enzyme activity in fibroblast extracts from heterozygotes. PCC activity in fibroblast extracts from controls and from obligate heterozygotes belonging to either the pcc $A$ or pcc $C$ complementation groups is shown in
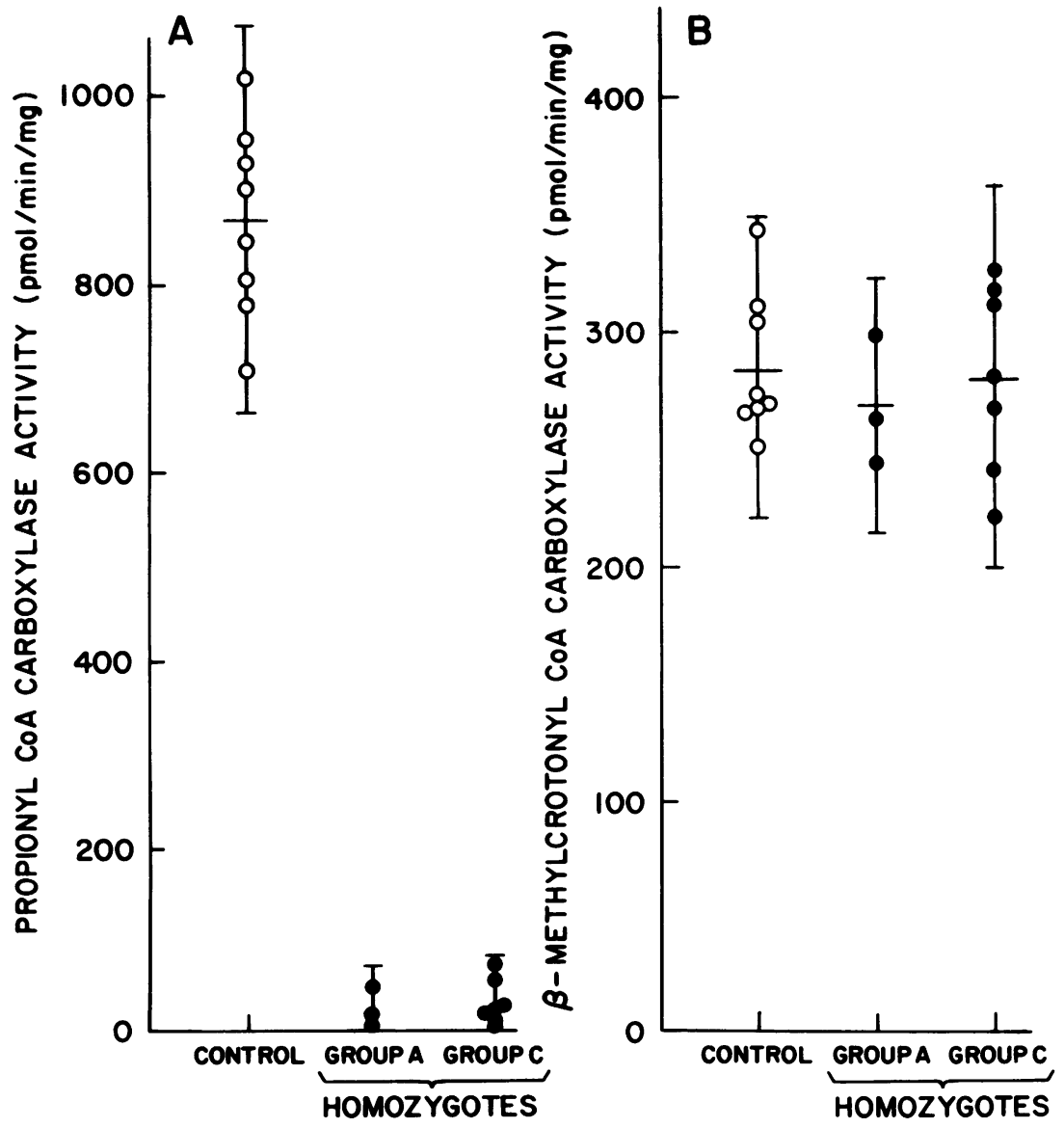

FIGURE 1 Comparison of PCC and $\beta$ MCC activities in fibroblast extracts from controls $(O)$ and homozygotes for PCC deficiency from the pcc $A$ and pcc $C$ complementation groups (O). In this and all subsequent figures, mean activities are shown as the longer transverse line with 2 SD above and below the mean shown by the capped solid vertical line. 

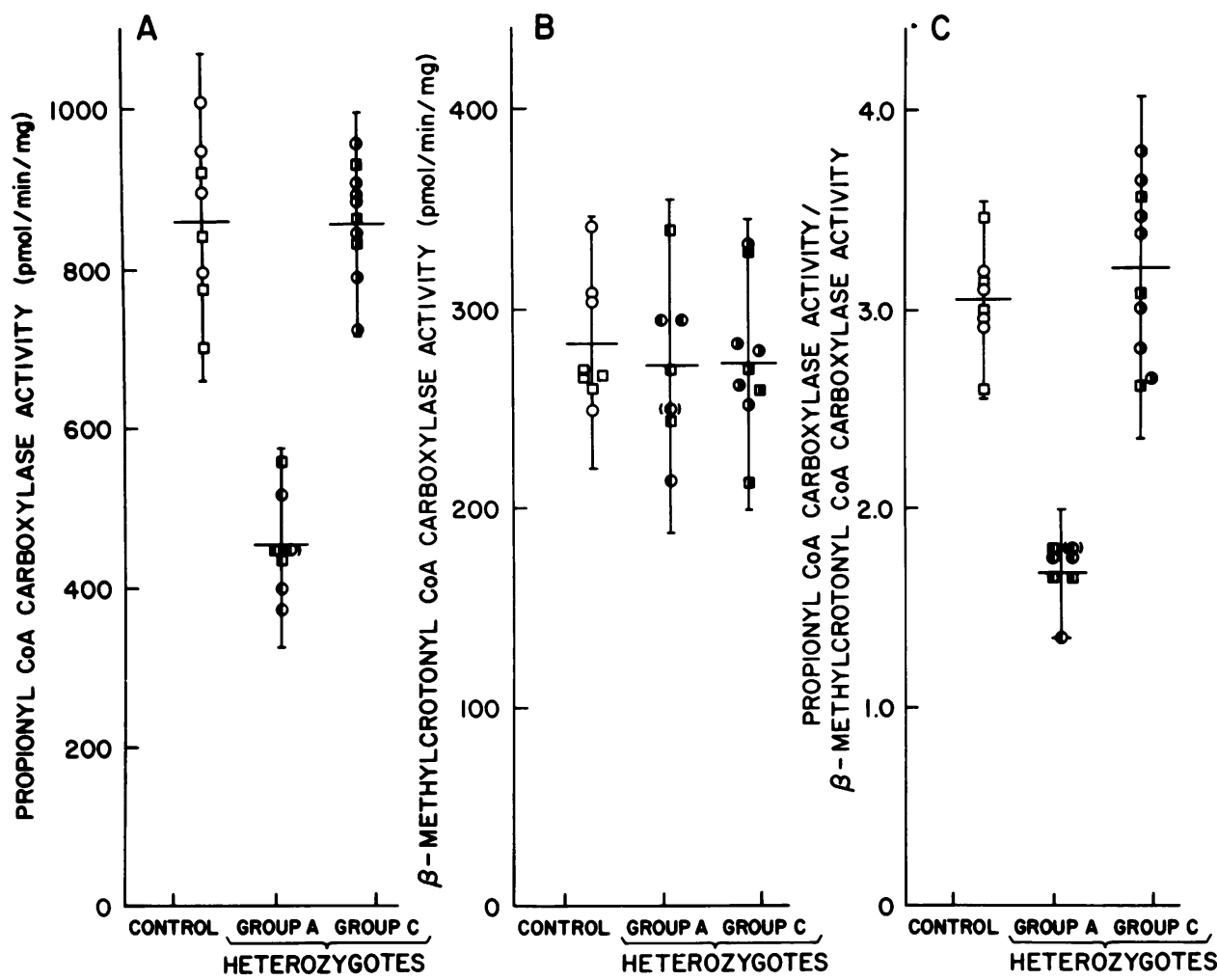

FIGURE 2 Comparison of PCC (A), $\beta$ MCC activities (B), and the ratio of PCC to $\beta$ MCC activities $(C)$ in fibroblast extracts from controls $(O \square)$ and obligate heterozygotes from pcc $A(O D)$ and pcc $C$ (О口) groups. Results from a sibling of a pcc A patient are shown in parentheses. Females are denoted by circles; males by squares. See legend to Fig. 1 for additional details.

Fig. 2A. Mean PCC specific activity in the six pcc A heterozygotes was $454 \pm 65 \mathrm{pmol} / \mathrm{min}$ per $\mathrm{mg}(52 \%$ of mean control); no overlap with control values was noted. The difference is highly significant statistically $(P<0.001)$. The mean specific activity in extracts from pcc $C$ heterozygotes $(851 \pm 66 \mathrm{pmol} / \mathrm{min}$ per $\mathrm{mg})$, however, was indistinguishable from that of controls. No overlap with pcc $A$ heterozygotes was found. All parents of pcc A homozygous patients had approximately half of control PCC activity, whereas all parents of pcc $C$ patients had PCC activity in the control range. Thus, in the eight families in which both parents were studied, we found no evidence for the existence of genetic compounds or double heterozygotes.

Because $\beta$ MCC activity in fibroblast extracts from pcc $A$ heterozygotes $(272 \pm 42 \mathrm{pmol} / \mathrm{min}$ per $\mathrm{mg})$ and pcc $C$ heterozygotes $(276 \pm 29 \mathrm{pmol} / \mathrm{min}$ per $\mathrm{mg}$ ) was indistinguishable from that of controls (Fig. 2B), trivial explanations for the observed interclass differences in PCC activity, such as those related to passage number or protein concentration, are excluded. The ratio of PCC activity to $\beta$ MCC activity (Fig. 2C) further demonstrates the difference between $p c c A$ heterozygotes and either controls or $p c c C$ heterozygotes. The mean ratio for $p c c$ A heterozygotes, $1.68 \pm 0.16$, is significantly lower than that for either controls or $p c c C$ heterozygotes $(P<0.001)$. Activity of glutamate dehydrogenase, a mitochondrial enzyme that does not require biotin, was comparable in fibroblast extracts from patients, parents, and controls (data not shown) and thus served as an additional reference enzyme.

PCC activity in leukocyte extracts from heterozygotes. We also measured PCC activity in extracts of peripheral blood leukocytes from controls and from heterozygotes (Fig. 3). The results in leukocytes paralleled those seen in skin fibroblast extracts. Mean specific activity in pcc $C$ heterozygotes $(321 \pm 28 \mathrm{pmol} /$ min per $\mathrm{mg})$ was similar to that of controls $(349 \pm 51$ $\mathrm{pmol} / \mathrm{min}$ per $\mathrm{mg}$ ); mean specific activity in pcc $A$ heterozygotes $(182 \pm 33 \mathrm{pmol} / \mathrm{min}$ per $\mathrm{mg}$ ) was again significantly reduced $(P<0.001)$ compared with either controls or $p c c C$ heterozygotes.

\section{DISCUSSION}

Heterozygotes for most autosomal recessively inherited disorders due to specific enzyme deficiencies have enzyme activities intermediate between those of 


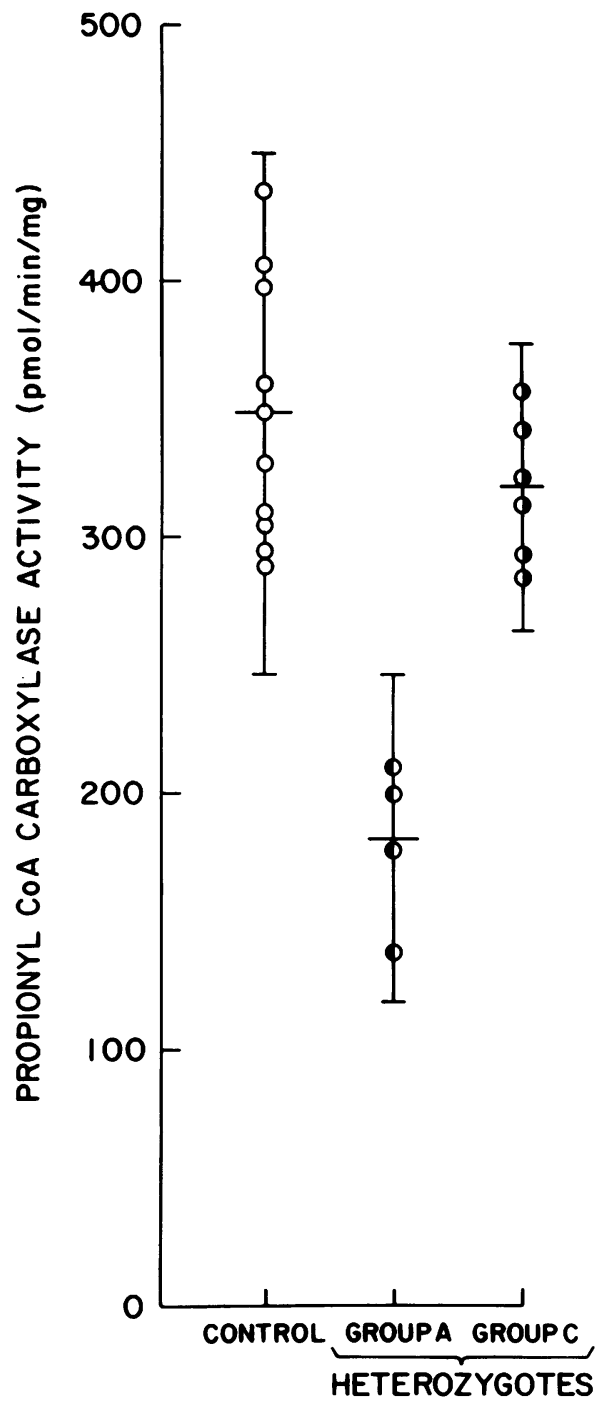

FIGURE 3 Comparison of PCC activity in extracts of peripheral blood leukocytes from controls $(O)$ and obligate

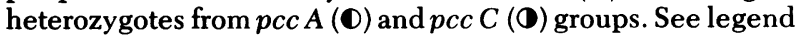
to Fig. 1 for additional details.

controls and homozygous affected individuals. This implies that there is generally no dosage compensation for autosomal mutations in human cells (12). In accord with this gene dosage model, heterozygotes for the pcc A mutation have PCC activity in extracts of fibroblasts and leukocytes approximately half of that found in controls. Surprisingly, all heterozygotes for the pcc $C$ mutation had normal PCC activity in the two tissues studied. The prominent difference in residual PCC activity demonstrated between carriers for these two distinct pcc mutations cannot be explained, as we stated above, by trivial differences in growth rate, passage number, or protein content of fibroblasts in culture. Moreover, because PCC assays with cell extracts from pcc $A$ and pcc $C$ homozygotes have shown that the $K_{m}$ s for all enzyme substrates are in the normal range (6), there is no reason to believe that the differences are simply a function of the in vitro assay conditions.

To our knowledge, there are only two other examples of autosomal recessively inherited enzyme deficiencies in man in which direct enzyme assay has yielded normal results in obligate carriers. Hamilton and Neel (13) reported that heterozygotes for one type of acatalasemia had normal catalase activities in erythrocyte extracts. In a second, more recent instance, some heterozygotes in a family containing a proband with severe adenosine deaminase deficiency had adenosine deaminase activities in erythrocytes indistinguishable from that of controls, whereas other heterozygotes within the same family had activities distinctly below the normal range (14). In neither instance was the mechanism of these unexpected findings provided.

Theoretically, the normal PCC activities in pcc $C$ heterozygotes could reflect expression of either regulatory or structural gene mutations. However, we have shown recently that mutant PCC in cells from either pcc $A$ or pcc $C$ homozygotes is more labile to heat and cold than is normal PCC (6). Furthermore, mutant enzyme from pcc A (but not pcc $C$ ) cells has a reduced affinity for potassium activation. Given this evidence for different structurally altered PCC molecules in each mutant class, and, hence, for structural gene mutations, there is little reason to invoke mechanisms concerning regulatory genetic elements.

Critical interpretation of the present results requires detailed knowledge of the structure of PCC. Crystalline pig heart PCC has a mol wt of $\cong 700,000$, and is composed of four identical protomers, each with a mol wt of $\cong 175,000$ (15). Although the subunit structure of PCC remains unclear, a recent preliminary observation suggests that protomers of the enzyme from bovine kidney are composed of two subunits of different molecular weight, the larger one containing the biotin moiety (16). Although human PCC has a molecular weight similar to that reported for the enzyme from animal tissues (17), it is not known whether the protomers of human PCC are composed of a single polypeptide subunit encoded by a single gene, or of two or more subunits encoded by multiple genes.

If we assume that the human PCC protomer is composed of a single polypeptide, that the native enzyme is tetrameric, that normal and mutant $p c c C$ protomers are produced in equal numbers, and that they can associate randomly with each other, five classes of tetramers can be posited. If all tetramers containing at least one normal protomer have full catalytic activity, then $15 / 16$ of the assembled tetramers would have normal activity and only 1/16 would be deficient. Heterozygotes with 15/16 of control PCC activity would likely be indistinguishable from controls. There is some pre- 
cedent for this model. In mouse $\times$ human somatic cell hybrids, detectable enzymatic activity was recovered in interspecific tetramers in which one or two inactive mutant subunits of human $\beta$-glucuronidase combined with normal mouse subunits (18). We find this explanation for our results with $p c c C$ heterozygotes unlikely for two reasons. First, the existence of at least two complementation groups among PCC-deficient lines suggests that the PCC protomer is composed of at least two different subunits, not one (5). Second, previous studies of 100 mammalian and human enzymes have revealed no enzymes with subunit mol wt $>130,000$, including the biotin-dependent enzyme, pyruvate carboxylase $(19,20)$. Thus a protomer with a mol wt of 175,000 seems unlikely.

Alternatively, our findings in pcc $C$ heterozygotes may be explained if we assume the following: $(a)$ that as seems likely, PCC protomers are composed of two nonidentical polypeptide subunits, $\alpha$ and $\beta ;(b)$ that the pcc $A$ complementation group reflects a mutation of the $\alpha$-subunit and the pcc $C$ group a mutation of the $\beta$-subunits; and $(c)$ that normal cells contain twice as many $\beta$ as $\alpha$-subunits either because $\beta$-subunits are synthesized more rapidly or degraded more slowly than their $\alpha$-counterparts. Then, reducing the number of normal $\beta$-subunits by half, as would occur in cells from a pcc $C$ heterozygote, would balance the number of normal $\alpha$ - and $\beta$-subunits, still yield a normal number of PCC protomers and, hence, normal PCC activity.

Regardless of the molecular mechanism, the present results demonstrate that measurement of PCC activity in tissues from obligate heterozygotes provides independent, confirmatory evidence for the existence of the two distinct mutant classes defined by classic complementation analyses with somatic cell heterokaryons (5). The failure to find a single family (of eight tested) in which a $p c c A$ heterozygote married a $p c c C$ heterozygote and produced an affected offspring is particularly noteworthy. Because there are no trivial explanations for this failure to identify symptomatic genetic compounds or double heterozygotes (such as a high incidence of consanguinity, or geographic and ethnic isolation), these results imply that in vivo (as well as in vitro) complementation is being observed in PCC deficiency.

Finally, these data emphasize that in certain disorders, such as PCC deficiency, measurement of enzyme activity will not identify all carriers in an unselected population. This conclusion must be borne in mind as we consider extension of carrier detection programs, such as those for Tay-Sachs and sickle cell disease, to other conditions.

\section{ACKNOWLEDGMENTS}

We wish to thank W. A. Fenton, Y. E. Hsia, and H. F. Willard for their helpful discussions, and Ms. R. Blunden for her valuable technical assistance. We also wish to thank Doctors I. K. Brandt, M. L. Cowger, D. Harris, A. Schwartz, and R. Wappner, for referring patients or providing us with cell lines, and Ms. M. Feldman and L. Herold for their secretarial assistance.

This work was supported by a research grant from the National Institutes of Health (AM 12579).

\section{REFERENCES}

1. Ando, T., and W. L. Nyhan. 1974. Propionic acidemia and the ketotic hyperglycinemia syndrome. In Heritable Disorders of Amino Acid Metabolism. W. L. Nyhan, editor. John Wiley \& Sons, New York. 37-80.

2. Rosenberg, L. E. 1978. Disorders of propionate, methylmalonate, and cobalamin metabolism. In The Metabolic Basis of Inherited Disease. J. B. Stanbury, J. B. Wyngaarden, and D. S. Fredrickson, editors. McGraw-Hill Book Co., New York. 4th edition. 411-429.

3. Hsia, Yu. E., K. Scully, and L. E. Rosenberg. 1971. Inherited propionyl-CoA carboxylase deficiency in "ketotic hyperglycinemia." J. Clin. Invest. 50: 127-130.

4. Gompertz, D., P. A. Goodey, H. Thom, G. Russell, A. W. Johnston, D. H. Mellor, M. W. MacLean, M. E. FergusonSmith, and M. A. Ferguson-Smith. 1975. Prenatal diagnosis and family studies in a case of propionicacidemia. Clin. Genet. 8: 244-250.

5. Gravel, R. A., F. K. Lam, K. J. Scully, and Yu. E. Hsia. 1977. Genetic complementation of propionyl CoA carboxylase deficiency in cultured fibroblasts. Am. J. Hum. Genet. 29: 378-388.

6. Wolf, B., Y. E. Hsia, and L. E. Rosenberg. 1978. Biochemical differences between mutant propionyl CoA carboxylases from two complementation groups. Am.J. Hum. Genet. In press.

7. Willard, H. F., I. S. Mellman, and L. E. Rosenberg. 1978. Genetic complementation among inherited deficiencies of methylmalonyl CoA mutase activity: evidence for a new class of human cobalamin mutant. Am. J. Hum. Genet. 30: $1-13$.

8. Hsia, Y. E., and K. J. Scully. 1973. Propionic acidemia: diagnosis by enzyme assay in frozen leukocytes. $J$. Pediatr. 83: 625-628.

9. Weyler, W., L. Sweetman, D. C. Maggio, and W. L. Nyhan 1977. Deficiency of propionyl-CoA carboxylase and $\beta$ methylcrotonyl-CoA carboxylase in a patient with methylcrotonylglycinuria. Clin. Chim. Acta. 76: 321-328.

10. Beaufay, H., D. S. Bendall, P. Baudhuin, and C. De Duve. 1959. Tissue fractionation studies. XII. Intracellular distribution of some dehydrogenases, alkaline deoxyribonuclease, and iron in rat-liver tissue. Biochem. J. 73: 623-628.

11. Lowry, O. H., N. J. Rosebrough, A. L. Farr, and R. J. Randall. 1951. Protein measurement with the Folin phenol reagent. J. Biol. Chem. 193: 265-275.

12. Harris, H. 1975. The Principles of Human Biochemical Genetics. North-Holland Publishing Co., Amsterdam, 235.

13. Hamilton, H. B., and J. V. Neel. 1963. Genetic heterogeneity in human acatalasia. Am. J. Hum. Genet. 15: 408419.

14. Chen, S-H., C. R. Scott, E. R. Giblett, and A. S. Levin. 1977. Adenosine deaminase deficiency: another family with a "silent" ADA allele and normal ADA activity in two heterozygotes. Am. J. Hum. Genet. 29: 642-644.

15. Kaziro, Y., S. Ochoa, R. C. Warner, and J-Y. Chen. 1961. Metabolism of propionic acid in animal tissues. VIII. 
Crystalline propionyl carboxylase. J. Biol. Chem. 236: 1917-1923.

16. Lau, E. P., L. Munson, B. C. Cochran, and R. R. Fall. 1978. Bipartite substructures of bovine kidney propionyl-CoA and 3-methylcrotonyl-CoA carboxylases. Fed. Proc. 37: 1574.

17. Hsia, Y. E., K. J. Scully, and L. E. Rosenberg. 1978. Human propionyl CoA carboxylase: some properties of the partially purified enzyme in fibroblasts from controls and patients with propionic acidemia. Pediatr. Res. In press.

18. Chern, C. J. 1977. Detection of active heteropolymeric $\beta$-glucuronidase in hybrids between mouse cells and human fibroblasts with $\beta$-glucuronidase deficiency. Proc. Natl. Acad. Sci. U. S. A. 74: 2948-2952.

19. Hopkinson, D. A., Y. H. Edwards, and H. Harris. 1976. The distribution of subunit numbers and subunit sizes of enzymes: a study of the products of 100 human gene loci. Ann. Hum. Genet. 39: 383-411.

20. Barden, R. E., B. L. Taylor, F. Isohashi, W. H. Frey, II, G. Zander, J. C. Lee, and M. F. Utter. 1975. Structural properties of pyruvate carboxylases from chicken liver and other sources. Proc. Natl. Acad. Sci. U. S. A. 72: $4308-4312$. 